\title{
The longitudinal association of cognitive appraisals and coping strategies with physical functioning in older adults with joint pain and comorbidity: a cohort study
}

Lotte A. H. Hermsen ${ }^{1}$, Johannes C. van der Wouden ${ }^{1 *}$, Stephanie S. Leone ${ }^{1,2}$, Martin Smalbrugge ${ }^{1}$, Henriëtte E. van der Horst ${ }^{1}$ and Joost Dekker ${ }^{3,4}$

\begin{abstract}
Background: Substantial variation exists in physical functioning (PF) among patients with comparable pain severity, which may be partly explained by underlying psychological processes, like cognitive appraisal of pain and coping with pain. It remains unclear to what extent such determinants contribute to changes in PF over time, especially in older populations. Therefore, we examined longitudinal associations of cognitive appraisals and coping strategies with PF, in older adults with joint pain and comorbidity.

Methods: A prospective cohort study among 407 older adults with joint pain and comorbidity provided data over 18 months, with 6 month time-intervals. We measured PF (RAND-36), five cognitive appraisals (consequences, concerns, emotional representations, self-efficacy, catastrophizing), four coping strategies (ignoring pain, positive self-statement, increasing activity levels, activity avoidance) and three time-dependent covariates; pain intensity, anxiety and depressive symptoms. Longitudinal associations were analyzed with Generalized Estimated Equations (GEE), by testing auto-regressive models, adjusted for covariates.

Results: More negative thoughts about consequences of pain $(\beta=-0.54,95 \% \mathrm{Cl}=-1.02 ;-0.06)$, more catastrophizing $(\beta=-0.67,95 \% \mathrm{Cl}=-1.26 ;-0.07)$ and more activity avoidance $(\beta=-0.32,95 \% \mathrm{Cl}=-0.57 ;-0.08)$ were significantly associated with subsequent deterioration in PF, whereas higher perceived self-efficacy $(\beta=0.22,95 \%$ $\mathrm{Cl}=0.12 ; 0.31$ ) was associated with subsequent improvement in PF. Neither concerns, emotional representations, ignoring pain, positive self-statement nor increasing activity levels were longitudinally related to PF.

Conclusions: More negative thoughts about consequences of pain, more catastrophizing and more activity avoidance contributed to deteriorated PF, whereas higher perceived self-efficacy contributed to improved PF. This knowledge may contribute to future management of functional limitations in older adults with joint pain and comorbidity.
\end{abstract}

Keywords: Longitudinal, Physical functioning, Pain, Older adults, Psychosocial, Coping, Appraisal, Musculoskeletal

\footnotetext{
* Correspondence: j.vanderwouden@vumc.nl

${ }^{1}$ Department of General Practice and Elderly Care Medicine, EMGO+ Institute

for Health and Care Research, VU University Medical Center, van der

Boechorststraat 7, 1081 BT Amsterdam, The Netherlands

Full list of author information is available at the end of the article
} 


\section{Background}

Joint pain is the leading cause of functional limitations in older populations. Low back pain, neck pain, and osteoarthritis are among the top 20 causes of disability [1]. In patients over 65 of age, self-reported pain of hip and knee show prevalence rates of 25-30\% [2]. However, it is not self-evident that all individuals with severe pain experience poor physical functioning (PF); in groups of people with comparable pain severity, some improve, some remain stable and others (gradually) deteriorate in PF [3, 4]. This variation may be partly explained by underlying psychological processes $[5,6]$. The transactional model of stress provides an interpretation of two complex psychological processes of responding to pain: appraisal and coping [6]. The first process 'appraisal' refers to an initial judgement of the pain, thus a personal evaluation whether pain is irrelevant or stressful. It also refers to individual beliefs about coping options and their effectiveness [6]. The appraisal process can result in negative (e.g., catastrophizing) or positive (e.g., higher perceived self-efficacy) cognitive appraisals of pain, which subsequently can influence one's ability to cope with pain. Coping is the second process that refers to the actual management of pain, in order to minimize the impact of pain on functioning. This can refer to cognitive coping strategies; i.e., changing the way one thinks/feels about the stressful situation (e.g., ignoring pain) or to behavioural coping strategies; i.e., actually changing the way one handles/deals with the situation (e.g., increasing activities).

A wide range of cognitive appraisals and coping strategies have been studied in relation to pain-related functional limitations. Most relevant contributors to poor PF seem to be more negative thoughts about consequences of pain, concerns and emotional representations regarding pain [7-9], lower perceived self-efficacy [10-12], more catastrophizing [13-15] and more activity avoidance [15-17]. The evidence about the relation of other coping strategies, like ignoring pain, positive self-statement or increasing activity level, with PF is inconsistent $[5,13]$. Several studies have a cross-sectional design which precludes inferences about cause and effect $[10,15,16]$. Moreover, earlier longitudinal studies primarily focused on the presence of these determinants at baseline in relation to changes in PF over time $[3,5,8,11-14,17]$, while longitudinal studies with repeated measures can test temporality; the determinant (appraisal/coping) precedes the outcome in time (PF) and make it possible to study the time-varying nature of the determinants in relation to changes in PF. Also, many studies focused on populations with specific conditions, such as osteoarthritis (OA) or rheumatic diseases or only on particular pain sites, like pain in the hip or knee $[3,8,9,12,13,15,16]$, while many older adults suffer from multiple joint pain sites [18]. Additionally, some studies were performed in middle-aged groups $[9,11,16,17]$ or did not take into account the presence of comorbidity, which is highly prevalent in older populations [19] and may influence the relation of cognitive appraisals and coping strategies with PF.

Therefore, the aim of this study was to examine the longitudinal association of five cognitive appraisals and four coping strategies with PF, in older adults with (multiple) joint pain and comorbidity.

\section{Methods}

We used data from a prospective cohort study that included 407 participants of 65 years or older, with more than two recorded chronic diseases in the medical file of the general practitioner and self-reported joint pain on most days in the past month in at least one joint pain sites: neck, back, shoulder, elbow, hand/wrist, hip, knee or ankle/foot. Participants were excluded if they lived in a nursing home, resided outside the research area for a prolonged period of time, had a life threatening illness, suffered from cognitive impairments or had insufficient knowledge of the Dutch language. Participants were recruited from 22 general practices in the region of Amsterdam and Haarlem, including both urbanized and rural areas, in the period November 2010-September 2011. More details about the study design have been previously published [20]. Data was collected at baseline and at 6,12 and 18 months follow-up.

The Medical Ethics Committee of the VU Medical Center Amsterdam approved the study protocol (\#2010/43) and written informed consent was obtained from all participants.

\section{Physical functioning}

Physical functioning was measured with the RAND-36 PF subscale, which asks about limitations in ten activities; vigorous activities, moderate activities, lift/carry groceries, climb several flights, climb one flight, bend/kneel, walk 1 $\mathrm{km}$, walk $0.5 \mathrm{~km}$, walk $100 \mathrm{~m}$, bath/dress [21]. Items were scored on an ordinal scale (severe, some, no limitations), recoded, summed into scale scores and transformed to a 0-100 score; lower score reflects more limitations. The RAND-36 has been proven to be reliable and valid [21].

\section{Appraisal and coping}

Based on the strongest evidence of being associated with $\mathrm{PF}$, we selected five cognitive appraisals: concerns, consequences, emotional representations, self-efficacy, catastrophizing and four coping strategies: ignoring pain, positive self-statement, increasing activity levels, activity avoidance [5,8-16]. Fig. 1 illustrates the location of the selected determinants in the transactional model of stress. We measured three appraisals with the Brief Illness Perception Questionnaire (B-IPQ) and replaced illness for joint pain: consequences -expected outcome of joint pain-; concerns -concerns because of joint pain-; emotional 


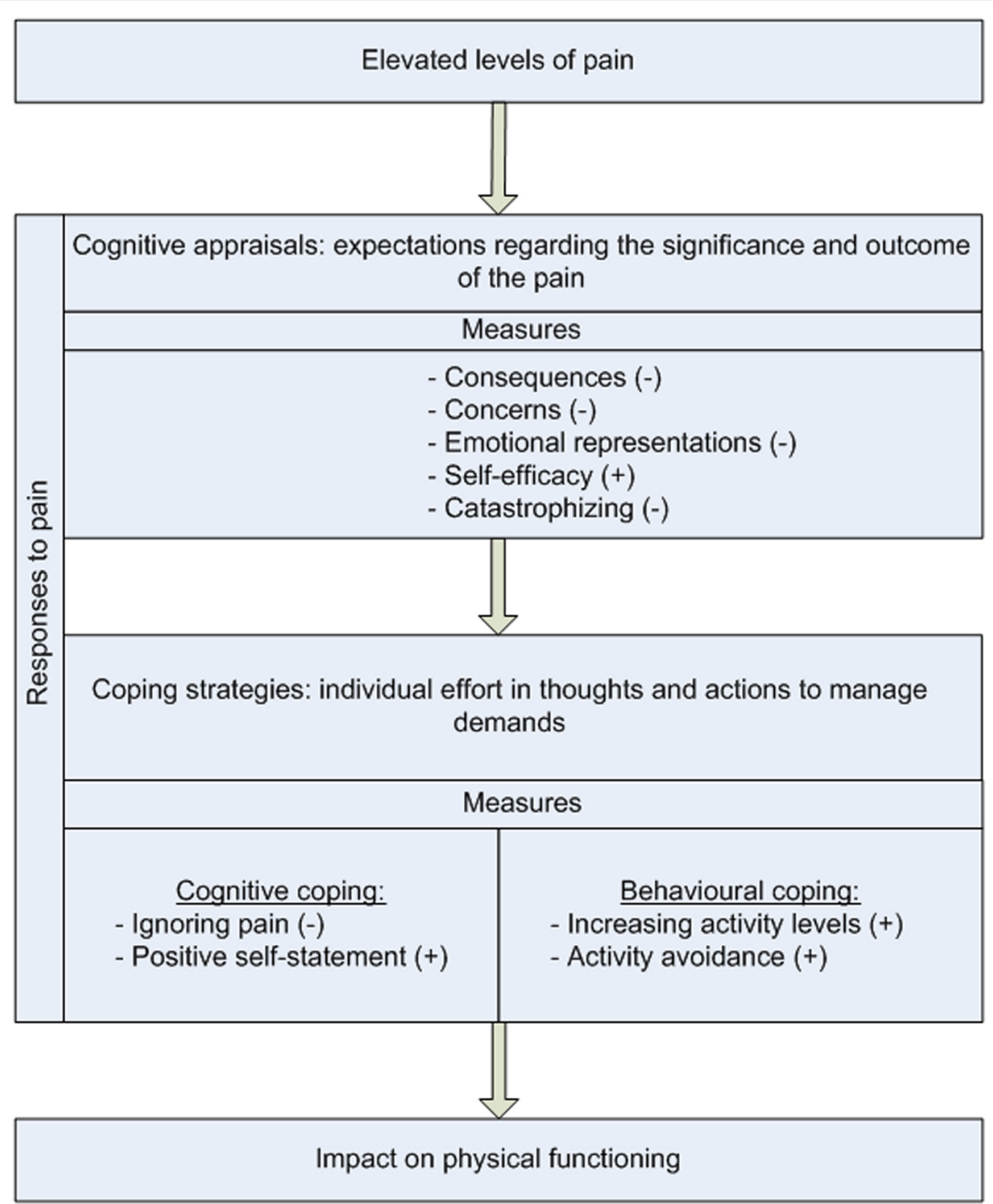

Fig. 1 Location of the selected cognitive appraisals and coping strategies in the transactional model of stress [5]. (-) negative influence on outcome; (+) positive influence on outcome

representations -anger, fear and distress because of joint pain- [21]; individual rating scale 0-10; a higher score reflecting more negative perceptions of joint pain. The B-IPQ has been shown to be reliable and valid [22]. The fourth appraisal self-efficacy was measured with the short form 6-item Arthritis Self Efficacy Scale (ASES) [23]. Since the dimensionality of the ASES remains unclear [24], we performed exploratory factor analysis and found evidence for a one-factor model with high factor loadings on the 6 items (0.59 to 0.84) (data not shown). Therefore, we calculated a total score, in which we summed the individual scores (1-11 rating scale) on the 6-items; score range 6-60; a higher score indicating more perceived selfefficacy. It has been debated whether catastrophizing is an appraisal or coping strategy [25]. Based on the available evidence, we categorized catastrophizing as an appraisal [25]. This fifth appraisal, catastrophizing, was measured with the short form Coping Strategy Questionnaire (CSQ), which uses two items to assess catastrophizing. Three coping strategies were also measured with the short form CSQ: ignoring pain, positive self-statement and increasing activity levels [23], again two items per coping strategy. Each individual item was scored on a 0-6 rating scale and a mean score of the two items was calculated; a higher score indicating more catastrophizing or more frequent use of the coping strategy. The fourth coping strategy activity avoidance was measured with the 5 -item resting subscale of the pain coping inventory (PCI) [26]; score range 5-20; a higher score indicating more painrelated activity avoidance. 


\section{Covariates}

Age, gender and education data were derived from the baseline questionnaire. Pain intensity, anxiety and depression were measured at all four time points. The Chronic Pain Grade (CPG) measured pain intensity based on the mean of the average, worst and present pain on a $0-100$ rating scale [27]; a higher score indicating more severe pain. The 14-item Hospital Anxiety and Depression Scale (HADS) measured anxiety symptoms with 7 items (score range 0-21) and depressive symptoms with 7 items (score range 0-21); a higher score indicating more symptoms [28].

\section{Statistical analysis}

Descriptive statistics characterised the study population. Mean scores and standard deviations (SD) of the cognitive appraisals, coping strategies and $\mathrm{PF}$ at baseline and after 6,12 and 18 months are presented. The longitudinal associations of the cognitive appraisals and coping strategies with PF were analyzed with Generalized Estimated Equations (GEE), which takes into account dependency between repeated measures. The technique allows all participants to be included in the analysis, regardless of missing data. We tested an auto-regressive (AR) model (see Fig. 2). Whereas the standard GEE model provides regression coefficients that combine between-participant and within-participant relationships, the AR model yields a better understanding of the actual longitudinal relationships, as the cross-sectional relationships (between-participant relationship) are removed [29]. It investigates whether absolute scores on a particular appraisal or coping strategy (independent variable) at one time point $(t)$ is associated with a higher scores on $\mathrm{PF}$ (dependent variable) at the next time point $(t+1)$, in which the model corrects for previous PF score. Thus, we explored if a change in PF would be a consequence of either previous appraisal or coping score and previous PF score. An independent working correlation structure was used [29] and the regression coefficients with $95 \%$ confidence intervals are presented for the unadjusted (only time included) and the adjusted model: corrected for time, time-independent covariates age, gender, education and time-dependent covariates pain intensity, anxiety and depressive symptoms. Additionally, we tested possible interaction effects of age, gender and pain intensity on the unadjusted relation between cognitive appraisals, coping strategies and PF. A $p$-value $<0.10$ indicated effect modification. Data were analysed using SPSS version 20.0.

\section{Results}

The recruitment process resulted in a total of 407 participants who fulfilled our inclusion criteria, and completed baseline assessments (see Fig. 3 for a flowchart). In the group of eligible patients, no significant differences were found between participants and non-participants regarding gender and age. However, non-participants were diagnosed with fewer chronic diseases (2.59 vs. 2.74, $t=-2.12, p=0.04)$, reported pain in fewer joint pain sites (3.67 vs. $4.19, t=-3.18, p<0.01)$ and lower pain severity (64.9 vs. $68.4, t=-2.06, p=0.01)$. Of the included 407 participants, 317 completed the study (77.9\%). The drop out percentages were $9.1 \%$ after 6 months, $7.8 \%$ after 12 months and $6.5 \%$ after 18 months. The most important reasons for drop out were death and deteriorated health. There were no differences in gender, number of chronic diseases, number of joint pain sites and pain intensity between the completers (participants with all four measures) and non-completers (drop outs, thus participant with incomplete data). However, compared to completers, non-completers were older (79.7 vs. 76.1, $t=4.94$, $p<0.001)$, lower educated $\left(\chi^{2}=21.09, p<0.001\right)$, reported poorer PF (38.1 vs. $51.6, t=-4.89, p<0.001)$, more anxiety (6.0 vs. $4.8, t=2.65, p=0.008$ ) and more depressive symptoms (6.7 vs. 5.1, $t=4.01, p<0.001)$. The characteristics of the study population are shown in Table 1 .

Scores of cognitive appraisals, coping strategies and PF were fairly stable over time (Table 2). The results of the AR models tested with GEE are presented in Table 3. The unadjusted and adjusted models showed

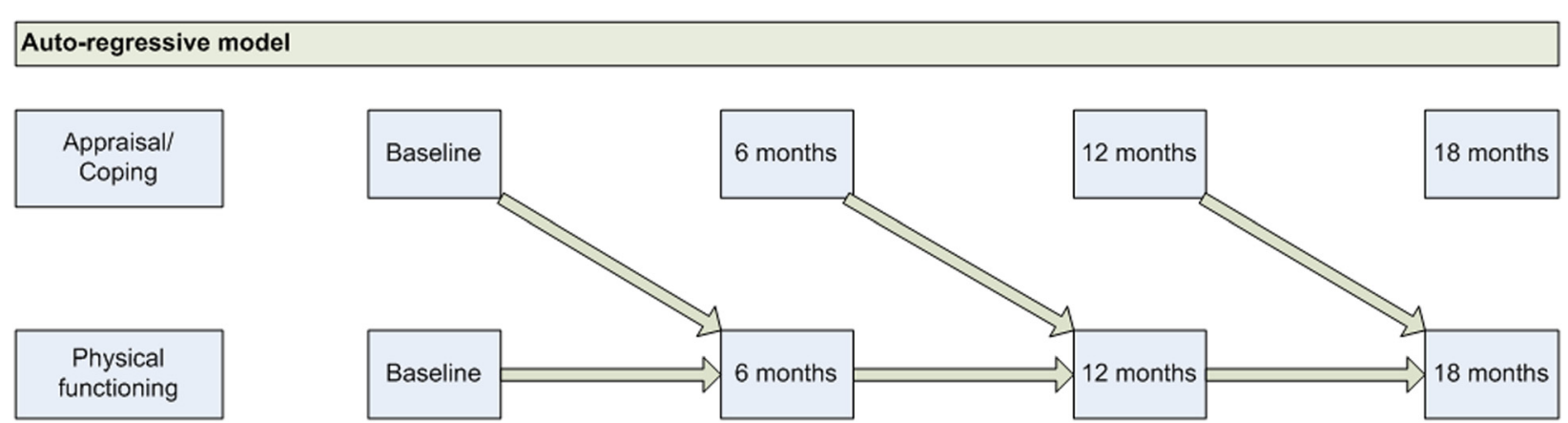

Fig. 2 Illustration of the auto-regressive model that we used to study the longitudinal relationship of cognitive appraisals and coping strategies with physical functioning 


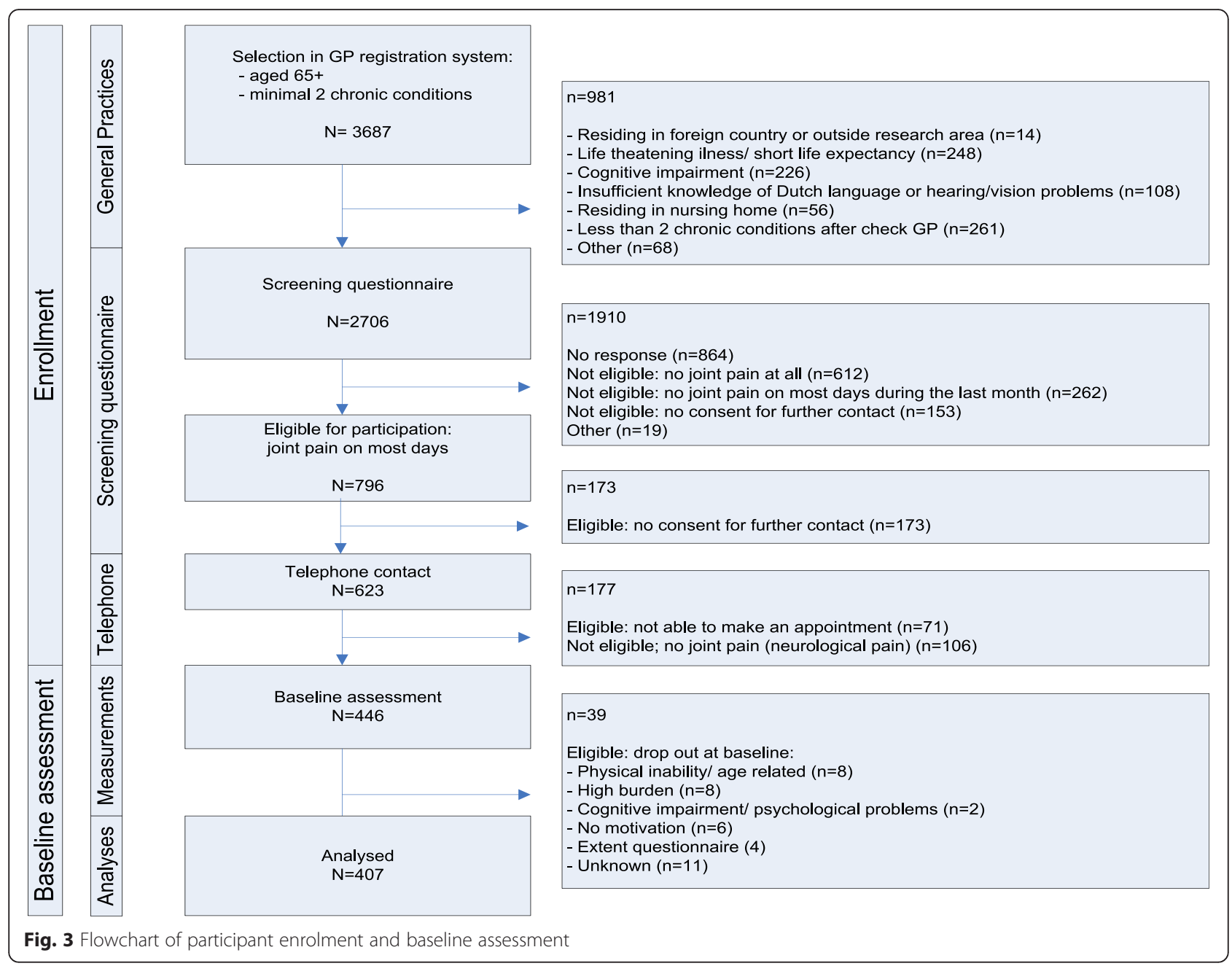

similar results, except for the relation between concerns about pain and deterioration in $\mathrm{PF}$, which was no longer significant in the adjusted AR model. In the adjusted models, more negative thoughts about the consequences of joint pain $(\beta=-0.54,95 \% \mathrm{CI}=-1.02 ;-0.06)$, more catastrophizing $(\beta=-0.67,95 \% \mathrm{CI}=-1.26 ;-0.07)$ and more activity avoidance $(\beta=-0.32,95 \% \mathrm{CI}=-0.57 ;-0.08)$ were significantly associated with subsequent deterioration in $\mathrm{PF}$, whereas higher perceived self-efficacy $(\beta=0.22,95 \% \mathrm{CI}=$ 0.12 ; 0.31 ) was significantly associated with subsequent improvement in PF. For clarification, the regression coefficient for activity avoidance can be interpreted as follows: a one point higher score in avoidance was related to a 0.36 deterioration in $\mathrm{PF}$ at the following time point.

We separately explored the interaction effect between each of the cognitive appraisals and coping strategies on the one hand, and age, gender and pain intensity on the other hand. Results with a $p$-value below our predefined level of 0.10 showed that the relation between selfefficacy and PF was weaker in the higher aged participants $(\beta=-0.009, P=0.051)$. In contrast, the relation between activity avoidance and PF was stronger in higher aged participants $(\beta=0.029, P=0.099)$ and in participants with higher pain intensities $(\beta=0.009, P=0.070)$.

\section{Discussion}

We aimed to shed more light on the longitudinal associations of cognitive appraisals and coping strategies with PF in older adults with joint pain and comorbidity in this study. More negative thoughts about consequences of joint pain, more catastrophizing and more activity avoidance were significantly associated with subsequent deterioration in PF, whereas higher perceived self-efficacy was associated with subsequent improvement in PF. Notably, most coping strategies contributed only little to changes in PF in our study.

Our findings showed that especially cognitive appraisals of pain were related to changes in PF over time. Higher perceived self-efficacy was related to improvement in PF. This is in line with previous studies that also reported strong evidence for this relation, especially in groups of people that are challenged by deteriorating PF, because of 
Table 1 Baseline characteristics of the study population $(n=407)$

\begin{tabular}{ll}
\hline Gender: male, $n$ (\%) & $153(37.6)$ \\
Age, mean (SD) & $76.8(6.3)$ \\
Living arrangement: living together, $n$ (\%) & $242(59.5)$ \\
Highest education: $n$ (\%) & \\
$\quad$ Primary & $121(29.7)$ \\
$\quad$ Secondary & $199(48.9)$ \\
$\quad$ College/university & $87(21.4)$ \\
Number of chronic diseases: $\geq 3, n$ (\%) & $197(48.4)$ \\
Chronic diseases: top 3, $n$ (\%) & \\
$\quad$ Chronic ischemic heart disease, heart failure & $254(62.4)$ \\
Diabetes mellitus & $152(37.3)$ \\
Chronic respiratory disease & $113(27.8)$ \\
Number of joint pain sites, (1-8), mean (SD) & $4.0(1.9)$ \\
Worst pain site: top 3, $n$ (\%) & $109(27.2)$ \\
Back & $73(18.2)$ \\
Knee & $61(15.2)$ \\
Hand/wrist &
\end{tabular}

elevated pain levels [3, 10-12]. Probably, people with higher perceived self-efficacy are better able to manage their pain. This may lead to more successful coping to alleviate difficulties and subsequently to less functional limitations [10]. Also, our findings confirmed previous findings that catastrophizing is an important contributor of deterioration in PF [13-15]. Probably, exaggeration of the pain, feeling helpless and more negative evaluation of the ability to deal with pain, result in experiencing more functional limitations [25]. Although previous studies reported associations between negative illness beliefs and poorer PF in populations with low back pain, osteoarthritis and chronic widespread pain [7-9], these results were only partly supported in our study, as we only found a relation between more negative thoughts about the consequences of pain and deterioration in PF. Since most previous studies were conducted in substantially younger populations, our results suggest that the relation between the other two negative perceptions of pain and PF may be of less importance in older populations. Possibly, longer exposure time to pain and pain-related disabilities in older adults may lead to more acceptance, more adjustments and readdressing of expectations, and subsequently to fewer negative illness beliefs around pain.

Only the coping strategy activity avoidance was associated with poorer PF in our study. This well-known relation has often been often explained as follows: more avoidance of activities that induce pain can result in decreased muscle strength, leading to more joint problems, decreased stability and subsequently increased pain and more functional limitations [15-17]. Neither ignoring pain, nor positive self-statement, nor increasing activity levels was related to changes in PF in our sample. Previous studies also showed contradictory results for the

Table 2 Scores of the repeated measures over 18 months of cognitive appraisals, coping strategies, physical functioning, and time-dependent covariates

\begin{tabular}{|c|c|c|c|c|c|}
\hline & Score range & Baseline $(n=407)$ & 6 months $(n=364)$ & 12 months $(n=337)$ & 18 months $(n=319)$ \\
\hline \multicolumn{6}{|l|}{ Outcome } \\
\hline Physical functioning ${ }^{a}$ & $0-100$ & $48.7(25.8)$ & $50.1(27.4)$ & $49.8(27.5)$ & $49.5(27.4)$ \\
\hline \multicolumn{6}{|l|}{ Cognitive appraisals } \\
\hline Consequences & $0-10$ & $5.3(2.7)$ & $5.1(2.6)$ & $4.9(2.7)$ & $4.8(2.7)$ \\
\hline Concerns & $0-10$ & $5.1(3.7)$ & $5.5(3.0)$ & $5.2(3.1)$ & $5.2(3.0)$ \\
\hline Emotional representations & $0-10$ & $4.2(3.0)$ & $4.3(2.9)$ & $3.9(3.0)$ & $4.0(3.0)$ \\
\hline Self-efficacy ${ }^{a}$ & $6-60$ & $33.8(12.5)$ & $34.2(13.5)$ & $35.3(13.1)$ & $35.6(12.8)$ \\
\hline Catastrophizing & $0-6$ & $1.8(1.5)$ & $1.9(1.6)$ & $1.8(1.6)$ & $1.8(1.5)$ \\
\hline \multicolumn{6}{|l|}{ Cognitive coping } \\
\hline Ignoring pain ${ }^{a}$ & $0-6$ & $3.0(1.5)$ & $2.9(1.4)$ & $3.1(1.5)$ & $3.0(1.4)$ \\
\hline Positive self-statement $^{a}$ & $0-6$ & $3.5(1.8)$ & $3.5(1.8)$ & $3.4(1.8)$ & $3.4(1.7)$ \\
\hline \multicolumn{6}{|l|}{ Behavioural coping } \\
\hline Increasing activity levels ${ }^{\mathrm{a}}$ & $0-6$ & $3.4(1.7)$ & $3.3(1.6)$ & $3.4(1.6)$ & $3.3(1.6)$ \\
\hline Activity avoidance & $5-20$ & $12.2(3.5)$ & $12.1(3.6)$ & $12.2(3.6)$ & $12.0(3.6)$ \\
\hline \multicolumn{6}{|l|}{ Time-dependent covariates } \\
\hline Pain intensity & $0-100$ & $64.4(17.3)$ & $65.0(18.5)$ & $61.6(19.8)$ & $62.7(19.4)$ \\
\hline Anxiety symptoms & $0-21$ & $5.1(3.7)$ & $5.4(3.7)$ & $4.9(3.8)$ & $4.9(3.6)$ \\
\hline Depression symptoms & $0-21$ & $5.4(3.5)$ & $5.3(3.6)$ & $4.9(3.5)$ & $5.3(3.9)$ \\
\hline
\end{tabular}

ahigher score is positive 
Table 3 Longitudinal associations of cognitive appraisals and coping strategies with physical functioning; auto-regressive models tested with generalized estimated equations

\begin{tabular}{|c|c|c|c|c|c|}
\hline \multirow[b]{2}{*}{ Independent variable } & \multirow[t]{2}{*}{ Range } & \multicolumn{4}{|c|}{ Auto-regressive model } \\
\hline & & Model $1^{\mathrm{b}}$ & $P$-value & Model $2^{c}$ & $P$-value \\
\hline \multicolumn{6}{|l|}{ Cognitive appraisals } \\
\hline Consequences & $0-10$ & $-0.62(-1.03 ;-0.20)$ & 0.003 & $-0.54(-1.02 ;-0.06)$ & 0.027 \\
\hline Concerns & $0-10$ & $-0.30(-0.59 ;-0.01)$ & 0.045 & $-0.21(-0.54 ; 0.11)$ & 0.195 \\
\hline Emotional representations & $0-10$ & $-0.05(-0.33 ; 0.23)$ & 0.717 & $0.10(-0.25 ; 0.45)$ & 0.570 \\
\hline Self-efficacy ${ }^{a}$ & $6-60$ & $0.26(0.17 ; 0.36)$ & $<0.001$ & $0.22(0.12 ; 0.31)$ & $<0.001$ \\
\hline Catastrophizing & $0-6$ & $-0.87(-1.41 ;-0.33)$ & 0.002 & $-0.67(-1.26 ;-0.07)$ & 0.028 \\
\hline \multicolumn{6}{|l|}{ Cognitive coping } \\
\hline Ignoring pain ${ }^{a}$ & $0-6$ & $-0.02(-0.53 ; 0.50)$ & 0.953 & $-0.02(-0.54 ; 0.49)$ & 0.929 \\
\hline Positive self-statement $^{a}$ & $0-6$ & $0.10(-0.32 ; 0.53)$ & 0.632 & $-0.01(-0.44 ; 0.42)$ & 0.956 \\
\hline \multicolumn{6}{|l|}{ Behavioural coping } \\
\hline Increasing activity levels ${ }^{a}$ & $0-6$ & $0.06(-0.43 ; 0.54)$ & 0.823 & $0.05(-0.44 ; 0.54)$ & 0.839 \\
\hline Activity avoidance & $5-20$ & $-0.37(-0.62 ;-0.12)$ & 0.004 & $-0.32(-0.57 ;-0.08)$ & 0.008 \\
\hline
\end{tabular}

ahigher score is positive

${ }^{b}$ model $1=$ adjusted for time

cmodel 2 = adjusted for time and time-independent covariates: age, gender and education and time-dependent covariates: pain intensity, anxiety and depression

relationship between these three coping strategies and PF $[5,13]$.

Additional analyses showed a weaker relation between higher perceived self-efficacy and improvement in PF in older participants. As expected, older age was related to more reported functional limitations in our sample. Maybe the ageing process, elevated pain levels and disability lead to decreased confidence to control such alterations. On the other hand, it could be that older people are better capable to accept their disabilities and learn to redress expectations. Both explanations may explain the weakening of the relation between self-efficacy and PF [30]. In contrast, the relation between activity avoidance and PF was stronger in higher aged participants, with more elevated levels of pain. In our sample, higher age was related to more elevated levels of pain, multiple joint pain and more co-occurring chronic diseases, which all may lead to more avoidance of activities. Such passive coping can result in declined bodily condition and poorer PF.

\section{Strengths and limitations}

We focused on older adults with (multiple) joint pain and comorbidity; a highly prevalent combination that is underrepresented in literature. We used data from a large cohort. The repeated measures over time enabled us to use GEE to study the longitudinal relations between cognitive appraisals, coping strategies and PF. As such studies are scarce, our findings add important information to the existing knowledge about the temporality of these relations. Also, we used only validated instruments. Furthermore, we were able to show that the above mentioned relations remained present even after controlling for anxiety and depressive symptoms. This is important, as previous studies have shown that depression can result in lower levels of self-esteem, more pessimistic thoughts (catastrophizing), which subsequently can impede the motivation to apply coping strategies or facilitates the application of passive coping strategies, like avoiding activities [14, 25, 31].

Despite the identified relations, we should bear in mind that the changes in PF between baseline and 18 months follow-up were only small and that the clinical relevance may be limited. Possibly, this follow-up period is not sufficiently long to examine changes in PF. Although we corrected for many important covariates, we may have missed other factors that could influence the relation of cognitive appraisals and coping strategies with PF, like fatigue and sleeping problems. We recruited participants in general practice, and every Dutch citizen is listed with a general practitioner. This enhances the generalizability of our findings. However, our non-response analysis showed that the participants in our study were more impaired than non-participants. This may at least partly have been compensated by the fact that participants who were lost to follow-up showed poorer physical functioning.

A final limitation concerns cognitive function. Although we excluded potential participants who were known to have been diagnosed with dementia or severe cognitive impairment at baseline, we did not assess cognitive function. Declining cognitive function may have affected the responses to our questionnaires.

\section{Implications for future research}

Our findings may be helpful when designing interventions aimed at older adults with joint pain and comorbidity. 
Given the fact that pharmacological interventions can have multiple side effects with increasing age, a nonpharmacological approach would be most welcome. Advising people not to avoid activities because of joint pain, and stimulating positive cognitive appraisals are potential ingredients to be tested in future intervention studies. Given the fact that we found only small changes in physical functioning over a period of 18 months, future studies might consider a longer follow-up period. We however need to bear in mind that longitudinal research in an elderly population with impaired health is inevitably hampered by loss to follow-up.

\section{Conclusion}

This study showed that more negative thoughts about consequences of pain, more catastrophizing and more activity avoidance contributed to deteriorated PF, whereas higher perceived self-efficacy contributed to improved PF. This knowledge may contribute to future management of functional limitations in older adults with joint pain and comorbidity.

\section{Competing interests}

The authors declare that they have no competing interests.

\section{Authors' contributions}

LH, MS, SL, HvdH and JD designed the study. LH collected the data, performed the statistical analyses and wrote the paper. JvdW, MS, SL, HvdH and JD commented on drafts of the manuscript. All authors read and approved the final manuscript.

\section{Acknowledgements}

This study was funded by the Netherlands Organisation for Health Research and Development (ZonMw); Grant number: 313080301. The funders had no role in the design or publication of the manuscript.

\section{Author details}

'Department of General Practice and Elderly Care Medicine, EMGO+ Institute for Health and Care Research, VU University Medical Center, van der Boechorststraat 7, 1081 BT Amsterdam, The Netherlands. ${ }^{2}$ Public Mental Health, Netherlands Institute of Mental Health and Addiction, Utrecht, The Netherlands. ${ }^{3}$ Department of Rehabilitation Medicine, VU University Medical Center, Amsterdam, The Netherlands. ${ }^{4}$ Department of Psychiatry, VU University Medical Center, Amsterdam, The Netherlands.

\section{Received: 5 May 2015 Accepted: 24 January 2016}

Published online: 27 January 2016

\section{References}

1. Global Burden of Disease Study 2013 Collaborators. Global, regional, and national incidence, prevalence, and years lived with disability for 301 acute and chronic diseases and injuries in 188 countries, 1990-2013: a systematic analysis for the Global Burden of Disease Study 2013. Lancet. 2015:386:743-800.

2. Picavet HS, Hazes JM. Prevalence of self reported musculoskeletal diseases is high. Ann Rheum Dis. 2003;62:644-50.

3. Sharma L, Cahue S, Song J, Hayes K, Pai YC, Dunlop D. Physical functioning over three years in knee osteoarthritis - Role of psychosocial, local mechanical, and neuromuscular factors. Arthritis Rheum. 2003;48:3359-70.

4. van Dijk GM, Dekker J, Veenhof C, van den Ende $\mathrm{CH}$. Course of functional status and pain in osteoarthritis of the hip or knee: a systematic review of the literature. Arthritis Rheum. 2006;55:779-85.

5. Benyon K, Hill S, Zadurian N, Mallen C. Coping strategies and self-efficacy as predictors of outcome in osteoarthritis: a systematic review. Musculoskeletal Care. 2010:8:224-36.

6. Lazarus RS, Folkman S. Stress, Appraisal and Coping. New York: Springer; 1984
7. Foster NE, Bishop A, Thomas E, et al. Illness perceptions of low back pain patients in primary care: what are they, do they change and are they associated with outcome? Pain. 2008;136:177-87.

8. de Rooij A, van der Leeden M, Roorda LD, Steultjens MP, Dekker J. Predictors of outcome of multidisciplinary treatment in chronic widespread pain: an observational study. BMC Musculoskelet Disord. 2013;14:133.

9. Kaptein AA, Bijsterbosch J, Scharloo M, Hampson SE, Kroon HM, Kloppenburg M. Using the common sense model of illness perceptions to examine osteoarthritis change: A 6-year longitudinal study. Health Psychol. 2010;29:56-64.

10. Turner JA, Ersek M, Kemp C. Self-efficacy for managing pain is associated with disability, depression, and pain coping among retirement community residents with chronic pain. J Pain. 2005;6:471-9.

11. Denison E, Asenlof $P$, Lindberg P. Self-efficacy, fear avoidance, and pain intensity as predictors of disability in subacute and chronic musculoskeletal pain patients in primary health care. Pain. 2004;111:245-52.

12. Rejeski WJ, Miller ME, Foy C, Messier S, Rapp S. Self-efficacy and the progression of functional limitations and self-reported disability in older adults with knee pain. J Gerontol B Psychol Sci Soc Sci. 2001;56:S261-5

13. Alschuler K, Molton I, Jensen M, Riddle D. Prognostic value of coping strategies in a community-based sample of persons with chronic symptomatic knee osteoarthritis. Pain. 2013;154:2775-81.

14. Benyon K, Muller S, Hill S, Mallen C. Coping strategies as predictors of pain and disability in older people in primary care: a longitudinal study. BMC Fam Pract. 2013:14:67.

15. Somers TJ, Keefe FJ, Pells JJ, et al. Pain catastrophizing and pain-related fear in osteoarthritis patients: relationships to pain and disability. J Pain Symptom Manage. 2009;37:863-72.

16. Holla JF, van der Leeden M, Knol DL, et al. Avoidance of activities in early symptomatic knee osteoarthritis: results from the CHECK cohort. Ann Behav Med. 2012:44:33-42.

17. Steultjens MPM, Dekker J, Bijlsma JWJ. Coping, pain, and disability in osteoarthritis: a longitudinal study. J Rheumatol. 2001;28:1068-72.

18. Buchman AS, Shah RC, Leurgans SE, Boyle PA, Wilson RS, Bennett DA Musculoskeletal pain and incident disability in community-dwelling older adults. Arthritis Care Res. 2010:62:1287-93.

19. Reeuwijk KG, de Rooij M, van Dijk GM, Veenhof C, Steultjens MP, Dekker J. Osteoarthritis of the hip or knee: which coexisting disorders are disabling? Clin Rheumatol. 2010;29:739-47.

20. Hermsen LA, Leone SS, van der Windt DA, Smalbrugge M, Dekker J, van der Horst HE. Functional outcome in older adults with joint pain and comorbidity: design of a prospective cohort study. BMC Musculoskelet Disord. 2011;12:241.

21. van der Zee Kl, Sanderman R, Heyink JW, de Haes H. Psychometric qualities of the RAND 36-item health survey 1.0: a multidimensional measure of general health status. Int J Behav Med. 1996:3:104-22.

22. Broadbent E, Petrie KJ, Main J, Weinman J. The brief illness perception questionnaire. J Psychosom Res. 2006;60:631-7.

23. Jensen MP, Keefe FJ, Lefebvre JC, Romano JM, Turner JA. One- and two-item measures of pain beliefs and coping strategies. Pain. 2003;104:453-69.

24. Mielenz TJ, Edwards MC, Callahan LF. Item response theory analysis of two questionnaire measures of arthritis-related self-efficacy beliefs from communitybased US samples. Arthritis. 2010;2010:416796.

25. Sullivan MJ, Thom B, Haythomthwaite JA, et al. Theoretical perspectives on the relation between catastrophizing and pain. Clin J Pain. 2001;17:52-64.

26. Kraaimaat FW, Evers AWM. Pain-coping strategies in chronic pain patients: psychometric characteristics of the pain-coping inventory (PCI). Int J Behav Med. 2003;10:343-63.

27. Smith $\mathrm{BH}$, Penny $\mathrm{Kl}$, Purves $\mathrm{AM}$, et al. The chronic pain grade questionnaire: validation and reliability in postal research. Pain. 1997;71:141-7.

28. Spinhoven P, Ormel J, Sloekers PPA, Kempen GIJM, Speckens AEM, van Hemert AM. A validation study of the hospital anxiety and depression scale (HADS) in different groups of Dutch subjects. Psychol Med. 1997:27:363-70.

29. Twisk J. Applied longitudinal data analysis for epidemiology. A practical guide. Cambridge: Cambridge University Press; 2003.

30. Grime J, Richardson JC, Ong BN. Perceptions of joint pain and feeling well in older people who reported being healthy: a qualitative study. $\mathrm{Br} J \mathrm{Gen}$ Pract. 2010;60:597-603.

31. Sale JE, Gignac M, Hawker G. The relationship between disease symptoms, life events, coping and treatment, and depression among older adults with osteoarthritis. J Rheumatol. 2008;35:335-42. 